\title{
Properties of Broadband Non-Linear Lossy Materials Employed in the Electromagnetic Inverse Scattering during the Microchip Processing
}

\author{
Eytan Barouch $^{1}$, Stephen L. Knodle ${ }^{1^{*}}$, Steven A. Orszag ${ }^{2^{*}}$ \\ ${ }^{1}$ Department of Mechanical Engineering, Boston University, Boston, USA \\ ${ }^{2}$ Department of Mathematics, Yale University, New Haven, USA \\ Email: eytanbrch@gmail.com
}

Received May 20, 2013; revised June 20, 2013; accepted June 28, 2013

Copyright (C) 2013 Eytan Barouch et al. This is an open access article distributed under the Creative Commons Attribution License, which permits unrestricted use, distribution, and reproduction in any medium, provided the original work is properly cited.

\begin{abstract}
A method has been designed and implemented to describe the optical properties of lossy materials as a continuous functions of a finite wave length spectrum, needed in analysis of the Maxwell-Material equations. Measurements of the index of refraction $(N)$ and the absorption coefficient $(K)$ over a limited spectral range are used as input data. The (complex) permittivity function is then represented as a sum of five types of terms: a plasma term, a conductivity term, several Debye poles, several symmetric Lorentz poles as well as several asymmetric extended Lorentz ("XLorentz") poles. All these terms are particular solutions of the Lifshitz integral equation describing the dispersion relation of a monochromatic electromagnetic wave. This representation facilitates the numerical solution of broadband direct and the inverse scattering of electromagnetic waves for thin film stacks and composite physical structures, in particular those now employed by the microchip industry.
\end{abstract}

Keywords: Scattering; Inverse Scattering; Silicon-Material-Properties; Material Electric Permittivity

\section{Introduction}

The Maxwell-Material equations are given in its differential form by:

$$
\begin{gathered}
\nabla \cdot \boldsymbol{D}=\rho \\
\nabla \cdot \boldsymbol{B}=0 \\
\nabla \times \boldsymbol{E}=\boldsymbol{J}+\frac{\partial}{\partial t} \boldsymbol{H} \\
\nabla \times \boldsymbol{H}=-\frac{\partial}{\partial t} \boldsymbol{E}
\end{gathered}
$$

In Expressions (1a)-(1d), $D, E, B, H$ represent the displacement and electric fields, the inductive and magnetic fields and $\boldsymbol{J} \& \boldsymbol{\rho}$ are the current and charge density respectively.

With the continuing decrease in size and increase in density of nanodevices, including electronic microchips, there are increasing challenges in quality control and inspection and for improvements in manufacturing technology. Gone are the days when scanning electron mi-

*Deceased. This work has been completed after the untimely departure of Prof. S. A. Orszag and Dr S. L. Knodle. crographs (SEMs) could be routinely used in non-destructive and non-invasive ways to inspect structures built as part of manufacturing processes. Now it is necessary to develop methods which are far less intrusive, can be used on-line during the nanoscale manufacturing itself. An attractive technique to address these issues is the use of broad-band electromagnetic wave scattering by nanostructures. On one hand, if the wavelength of the electromagnetic waves is substantially larger than the structural scales of the device then such radiation is likely to be non-destructive. Yet, if the scattering of these waves is (nearly) uniquely determined by the structures and vice versa then scattering results can be used as a diagnostic tool to discern geometric details.

The goal of this paper is to provide a framework in which to compute both the direct and the inverse scattering of broad-band waves when propagating through complex lossy media. These computational methods and resulting simulations can then be used both to determine the scattering of waves by known structures (viz., direct scattering) and to infer the geometric structures and properties of devices known to produce a given (typically measured) spectrum (viz., inverse scattering). This is the 
first of a series of two papers to describe this new computational methodology in detail.

In this first paper, the goal is to introduce powerful methods to characterize the optical properties of lossy materials, a critical first step in formulating the Maxwell equations for wave propagation in such media. In the second paper, the computational methodology for direct scattering is described and applied to a variety of nanostructures. An efficient solution of the inverse problem is also given. In this latter problem, the scattering data is given and used to infer the geometric structures that produce it.

The focus here is on materials employed by the microchip industry, which include $\mathrm{Si}, \mathrm{SiO}_{2}, \mathrm{TiO}_{2}, \mathrm{Si}_{3} \mathrm{~N}_{4}$ etc. It must be emphasized that the direct and quite simple approach of passing cubic-splines through the measured values of the actual material refractive index $N$ and absorption coefficient $K$ is not effective since it lends itself to create wildly incorrect values outside the measurement domain. These incorrect values of $N$ and $K$ outside the measurement matrix have an overwhelming influence on both the direct and inverse scattering leading to quite poor results. The spurious results so produced are often a consequence of a pole in frequency space similar to the pole produced by perfectly absorbing layers (for the latter, see [1]).

Traditionally, $N$ for a given wavelength is expressed in terms of a principal-value integral of $K$ over the entire spectrum and vice-versa. These equations are known as the Kramers-Kronig [3] relations and are derived from the analyticity of $N+i K$. Explicitly, the Kramers-Kronig relations are given as:

$$
\begin{aligned}
\epsilon_{T} \text { ot }(\omega) & =N(\omega)+i K(\omega) \\
N(\omega) & =\frac{1}{\pi} P \int_{-\infty}^{\infty} \frac{K\left(\omega^{\prime}\right)}{\omega^{\prime}-\omega} d \omega^{\prime} \\
K(\omega) & =-\frac{1}{\pi} P \int_{-\infty}^{\infty} \frac{N\left(\omega^{\prime}\right)}{\omega^{\prime}-\omega} d \omega^{\prime}
\end{aligned}
$$

However, it is highly impractical to perform measurements over the entire spectrum, due to the limited capability of measuring instruments at widely different wavelengths. In practice a relatively narrow spectrum is measured, necessitating simultaneous determinations of both the refractive and absorption indices. As such, the data so obtained is discrete in nature, rendering limited validity to broad band scattering, since the permittivity is expressed as a continuous function in the Fourier transformed Maxwell-Material (MM) equations.

The main numerical scheme employed in the current approach is based on a combination of the Powell and Brent methods for optimization (see [2]) in which the assignment of the order of the parameters is correlated to their relative significance. Once one iteration is completed on all parameters, a vector is created and a direction of parameter movement is obtained. In this scheme, two controlling accuracy tolerances are employed to insure optimal results within a short computational time. The restriction on the ranges of the parameters imposed by physical principles, prohibits the use of a free-range optimization, and a standard map from infinite range to a finite domain is not practical. Therefore, the adopted method prevents the parameters to exit the allowed range by forcing the optimization scheme to rescale itself and stay within the physically acceptable domain. A particularly important restriction is that on the parameters of the plasma term. The plasma term can be easily decomposed into two partial fractions, one resembling a Debye pole and the other resembling a conductivity term. The term resembling a Debye term is subtracted from the conductivity term, and if one has not restricted the plasma parameters properly, one may end up with an unphysical negative conductivity.

Section 2 of this paper presents the basic material analysis of silicon and the approach to represent electrical properties of various materials employed in the integrated circuit (IC) industry. Section 3 discusses the results of several other essential materials used in the IC industry which can be very different in nature from silicon. For example, while PolySilicon is rather similar to crystalline silicon, it has quite different conductivity properties. In Paper II [3], numerical and computational considerations are discussed.

\section{Material Analysis}

The task of solving the Maxwell-Material equations is complicated by the fact that the displacement vector is proportional to the electric field, and the proportionaly function is a very complicated function of $N$ and $K$. Since the equations are continuous one must have a continuous representation of the permitivity function.

A typical material-file is normally expressed as a table of wavelength, $N$, and $K$. The material properties of significance here are the real and imaginary parts of the permittivity, the real and imaginary parts of the material-reflectivity and the efficiency spectrum (i.e., reflectivity) of a flat material. To obtain these properties for a given wavelength is straightforward, so if one wishes to develop numerical solutions for the so-called MM equations [3] in the frequency domain a standard input file is sufficient. However, the cost associated with the MM equation per wavelength is immense. If 200 spectral points are needed, the equations must be solved 200 times regardless of dimensions.

Since the numerical accuracy of the algorithms for solution of the Maxwell-Material equations is a direct func- 
tion of the wavelength, all equations are solved on the same grid, resulting in a very cumbersome and inefficient algorithm. On the other hand, the spatio-temporal MM equations provide the mechanism to obtain the scattered wave as a function of time and space, affording the computation of the scattered fields for all wavelengths using a sophisticated non-uniform grid fast Fourier transform (FFT).

The development and implementation of the spatiotemporal MM equations require more delicate analysis. The material response to an external electromagnetic field is usually expressed in terms of the material polarization vector, which appears as a nonlinear convolution term with the electric field. If the material is also magnetic, a similar convolution is required of the magnetic permittivity with the magnetic field. In other words, the price to be paid for the benefit of scattering results for arbitrarily dense spectra is dealing with considerably more complicated equations whose coefficients are not easily available.

What is necessary is the expression of the permittivity function as a continuous and differentiable function of the frequency. This task is performed routinely in the IC and material-science literature. One way to perform this task is to use Pade approximants and express the permittivity function as a rational function of the frequency. However, in most cases, even this method is not adequate. The fitted function must make physical sense as well as being computationally useful. Therefore, it must satisfy the Kramers-Kronig analytical relations and possess a Fourier transform that exists for all relevant frequencies and does not violate causality!

The safest way to proceed is to use the "Lifshitz Integral" [4] which contains the permittivity function inside its complicated dispersion relations. However, this is easier said than done. Analytic expressions of the "Lifshitz Integral" have eluded researchers for many years; this is discussed in detail in [5] who unfortunately used complex coefficients consistent with non-causal time domain permittivity. Accordingly, the availability of approximate solutions to the "Lifshitz Integral" for specific resonance frequencies suggests can be made by expressing the permittivity function of each material as a sum of all known approximate solutions. Each term satisfies all of the strict criteria discussed above. Explicitly the permittivity terms are given by:

$$
\begin{aligned}
\epsilon_{L k}(\omega) & =\frac{\left(\epsilon_{s k}-\epsilon_{\infty}\right) \omega_{s k}^{2}}{\omega_{s k}^{2}-\omega^{2}+i \omega v_{s k}} \\
\epsilon_{D j}(\omega) & =\frac{\left(\epsilon_{r j}-\epsilon_{\infty}\right)\left(\omega_{r j}\right)}{\omega+i v_{r j}}
\end{aligned}
$$

$$
\begin{gathered}
\epsilon_{p}(\omega)=1-\frac{\omega_{p}^{2}}{\omega\left(\omega+i v_{p}\right)}, \\
\epsilon_{X L i}(\omega)=\frac{\left(\epsilon_{x i}-\epsilon_{\infty}\right) \omega_{x i}^{2}+B_{x i} \omega}{\omega_{x i}^{2}-\omega^{2}+i \omega v_{x i}} \\
\epsilon_{T o t}(\omega)=\epsilon_{\infty}+\epsilon_{p}(\omega)+\frac{\sigma}{i \omega} \\
+\sum_{1}^{n} \epsilon_{L k}(\omega)+\sum_{1}^{m} \epsilon_{D j}(\text { omega })+\sum_{1}^{q} \epsilon_{X L i}(\omega) .
\end{gathered}
$$

where $\epsilon_{T o t}(\omega)$ represents the total permittivity, and $\epsilon_{L k}(\omega), \epsilon_{D j}(\omega), \epsilon_{p}(\omega), \epsilon_{X L i}(\omega)$ represent the Lorentz, Debye, plasma and soc-called linear "XLorentz terms" respectively, $\sigma$ is the electrical conductivity in a scalar form and $\omega$ is its corresponding frequency.

The estimation of the parameters of $\epsilon_{\text {Tot }}(\omega)$ in Equations (2) is a very elaborate process due to the physical restrictions mentioned above and also due to the fact that the standard cost (error) function in L2 norm is insufficient as well as inaccurate. To address these constraints, typically an input file of the three parameters wavelength, $N, K$ is first converted to an input file of seven parameters: wavelength, $N, K$, real and imaginary parts of the reflected amplitude, efficiency spectrum and the corresponding energy in $\mathrm{eV}$. Here the real and imaginary parts of the reflected wave are given explicitly by:

Reflected Amplitude:

$$
\frac{N-1-i K}{N+1-i K}
$$

Reflected real amplitude (Colunm 4):

$$
\frac{N^{2}-1+K^{2}}{(N+1)^{2}+K^{2}}
$$

Reflected imaginary amplitude (Colunm 5):

$$
\frac{-2 K}{(N+1)^{2}+K^{2}}
$$

Efficiency Spectrum (Colunm 6):

$$
\frac{(N-1)^{2}+K^{2}}{(N+1)^{2}+K^{2}}
$$

Wave energy (eV) (Column 7):

$$
\frac{(N-1)^{2}+K^{2}}{(N+1)^{2}+K^{2}}
$$

This 7-parameter input file, given partially for titanium di-oxide in Table 1, is inserted into optimization program, changing the cost function at each iteration. The result is a material file specifying the number of 
Table 1. Wave-length, $N$ and $K$, real and imaginary permittivity of the reflected field, efficiency Spectrum and energy (eV) of $\mathrm{TiO}_{2}$.

\begin{tabular}{|c|c|c|c|c|c|c|}
\hline WV & $N$ & K & $R e$ Refrac & Im Refrac & Efficiency & Energy \\
\hline $\mathrm{nm}$ & & & & & & $\mathrm{eV}$ \\
\hline 150 & 1.3963196 & 1.289804 & 0.352866 & -0.348316 & 0.245838 & 8.26667 \\
\hline 155 & 1.5010481 & 1.28182 & 0.366687 & -0.324581 & 0.239812 & 8 \\
\hline 160 & 1.591106 & 1.253217 & 0.37446 & -0.302549 & 0.231756 & 7.75 \\
\hline 165 & 1.6562853 & 1.209761 & 0.376413 & -0.284002 & 0.222344 & 7.51515 \\
\hline 170 & 1.697933 & 1.162129 & 0.37471 & -0.269342 & 0.212953 & 7.29412 \\
\hline 175 & 1.7187971 & 1.125732 & 0.372039 & -0.26001 & 0.201655 & 7.08571 \\
\hline 180 & 1.7282972 & 1.102633 & 0.369865 & -0.254667 & 0.201655 & 6.88889 \\
\hline 185 & 1.7331906 & 1.094197 & 0.369331 & -0.25248 & 0.200152 & 6.7027 \\
\hline 190 & 1.7331001 & 1.096765 & 0.369726 & -0.252923 & 0.200667 & 6.52632 \\
\hline 195 & 1.7445583 & 1.106205 & 0.373123 & -0.252665 & 0.20306 & 6.35897 \\
\hline 200 & 1.7511391 & 1.121965 & 0.376694 & -0.254196 & 0.206514 & 6.2 \\
\hline 205 & 1.7641943 & 1.144162 & 0.382294 & -0.255682 & 0.211522 & 6.04878 \\
\hline 210 & 1.7820588 & 1.1710085 & 0.389304 & -0.257051 & 0.217633 & 5.90476 \\
\hline 215 & 1.8099196 & 1.200139 & 0.398045 & -0.2571 & 0.22454 & 5.76744 \\
\hline 220 & 1.8445988 & 1.2302417 & 0.407698 & -0.256161 & 0.231836 & 5.63636 \\
\hline 225 & 1.8859383 & 1.2600557 & 0.417945 & -0.254136 & 0.239263 & 5.51111 \\
\hline 230 & 1.9343952 & 1.2876282 & 0.428476 & -0.250788 & 0.246486 & 5.3913 \\
\hline 235 & 1.984255 & 1.3141241 & 0.438665 & -0.247185 & 0.253527 & 5.2766 \\
\hline 240 & 2.0414818 & 1.3394241 & 0.44924 & -0.242547 & 0.260645 & 5.16667 \\
\hline 245 & 2.1080153 & 1.3599546 & 0.459909 & -0.236324 & 0.267336 & 5.06122 \\
\hline 250 & 2.1844189 & 1.3730942 & 0.470407 & -0.228356 & 0.273429 & 4.96 \\
\hline 255 & 2.2674101 & 1.3787183 & 0.480408 & -0.219247 & 0.278861 & 4.86275 \\
\hline 260 & 2.3548832 & 1.3745286 & 0.489541 & -0.20914 & 0.28339 & 4.76923 \\
\hline 265 & 2.4463011 & 1.3564226 & 0.497509 & -0.197774 & 0.28663 & 4.67925 \\
\hline 270 & 2.5374688 & 1.3235867 & 0.504055 & -0.185564 & 0.288505 & 4.59259 \\
\hline 275 & 2.6276192 & 1.277309 & 0.509488 & -0.172713 & 0.289407 & 4.50909 \\
\hline 280 & 2.721905 & 1.2117246 & 0.514139 & -0.15818 & 0.289359 & 4.42857 \\
\hline 285 & 2.8029651 & 1.1243056 & 0.516365 & -0.142981 & 0.287077 & 4.35088 \\
\hline
\end{tabular}

poles in each sum and the values of their corresponding parameters. An example of a 7-column file is given in the appendix for the crystalline titanium di-oxide file employed in this paper.

As an example, the silicon material file is displayed in Figure 1. The real and imaginary components of the measured and estimated values are compared with very good agreement. In particular, the elaborate structure of silicon challenges ones ability to fit it with simple elementary functions like the ones given in Equations (2).

In Figure 2, the computed efficiency spectrum of the optimized Si parameters is displayed as a function of wavelength. Note the complicated structure of this efficiency spectrum, particularly in the short wavelength domain. In Figure 3, the reflectivity of the optimized 


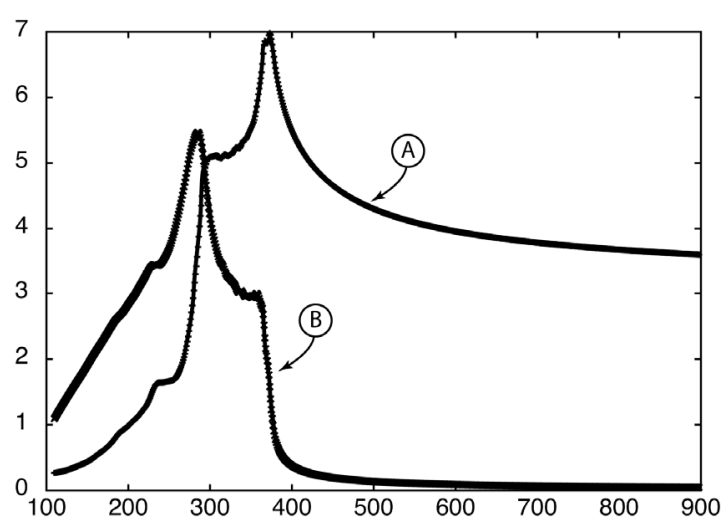

Figure 1. Real (A) and Imaginary(B) $\epsilon_{T o t}(\omega)$ using 34 Lorentz poles, 4 XLorentz poles and 3 Debye poles.

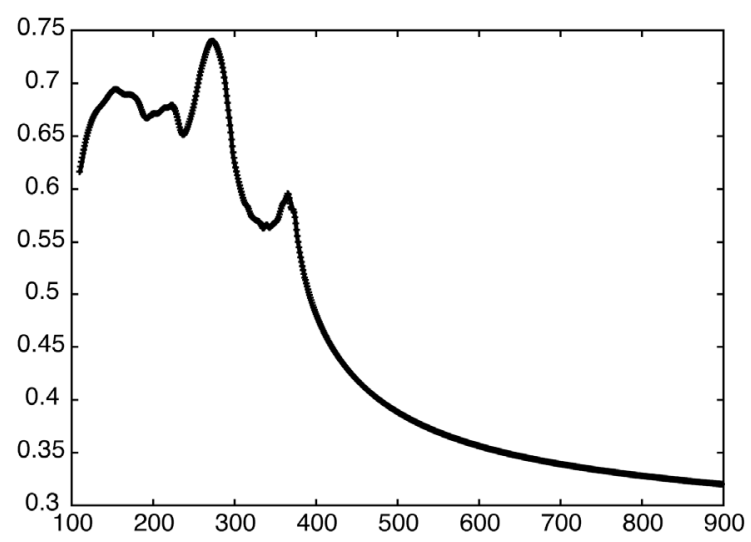

Figure 2. Efficiency Spectrum of planar Silicon. The graph represents the actual percentage of reflection vs. wavelength in $\mathrm{nm}$ for a pure silicon slab.

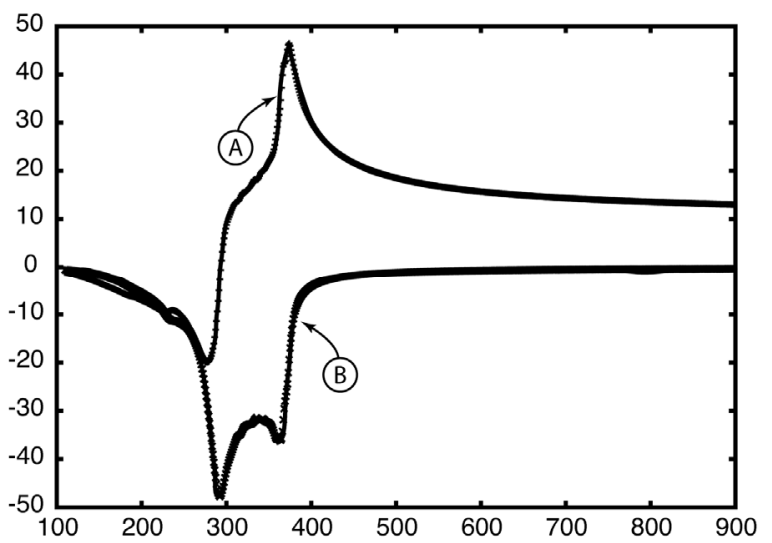

Figure 3. Real (A) and imaginary (B) coefficients of the reflected wave over a thick flat pure silicon.

silicon description is displayed. The two curves represent the real and imaginary amplitude of the relative reflected wave, given explicitly by

$\operatorname{Re}[(N-i K-1) /(N-i K+1)]$ and

$\operatorname{Im}[(N-i K-1) /(N-i K+1)]$ respectively. In Figure 4,

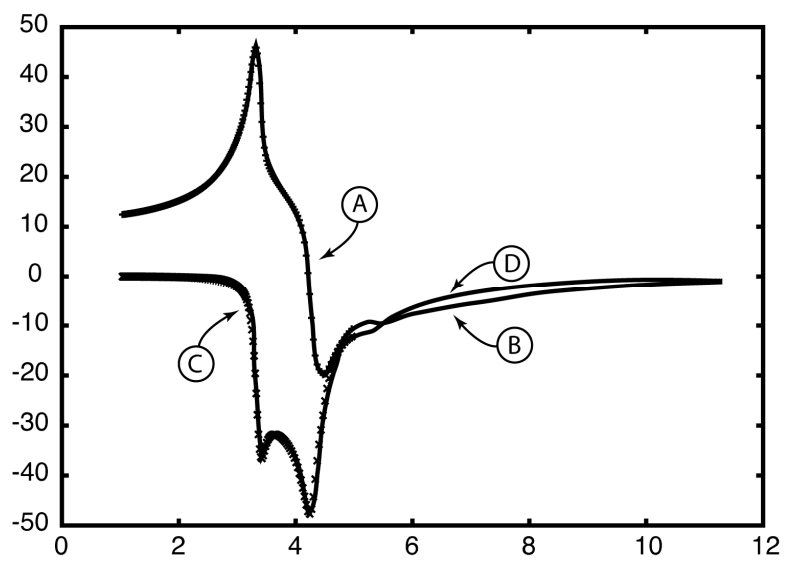

Figure 4. Real (A,D) and imaginary $(B, C)$ coefficients of the reflected wave over a thick flat pure silicon: A comparison of NIST data and the optimized material file vs. wave energy in $\mathrm{eV}$. As can be seen they are nearly indistinguishable.

a comparison is exhibited between estimated reflectivity and exact calculation from the data, using analytical expression for both real and imaginary components of the reflected wave. As can be seen, the comparison of the experimental data to the optimized continuous representation is very good. If one wishes, the methodology introduced here can deliver uniform machine accuracy.

\section{Other Materials Used in Microchip Processing}

The microchip industry needs the properties of several materials, in addition to crystalline silicon, during the photolithography process. The most critical lossy materials are $\mathrm{SiO}_{2}$ (which determines the transistor gate thickness), $\mathrm{Si}_{3} \mathrm{~N}_{4}$, PolySilicon (which is the actual material on top of the $\mathrm{SiO}_{2}$ gate) and $\mathrm{TiO}_{2}$. The photolithographic process is usually confined to a very narrow band width, which requires materials with very tight absorption and refraction properties. However, non-invasive investigation of the results must be performed with a considerably wider bandwidth in order to determine the actual shapes obtained from photolithography, thus assuring the fidelity to the original design. As explained above, the precise determination of $N$ and $K$ of materials from narrow band response is technically difficult, resulting in limited data which causes difficulties during optimization. The simple rule of thumb is that the more complicated the $N$ and $K$ structure of a material, the easier is to obtain reliable dense data vs. wavelength, as seen in the structure of the silicon and PolySilicon curves.

Here we present results for a verification of the materials mentioned. In each, the results contain the permittivity (left), the continuous representation of $N(\mathrm{~A})$ and $K(\mathrm{~B})$, and the real (A) and imaginary (B) part of the ratio 
of the reflected wave amplitude to the incident wave, given explicitly by $\operatorname{Re}[(N-i K-1) /(N-i K+1)]$ and $\operatorname{Im}[(N-i K-1) /(N-i K+1)]$ respectively. The material chosen here for a detailed presentation in titanium dioxide, which participate in the structure of every microchip. Again the $N$ and $K$ are displayed in Figure 5, the reflective real and imaginary amplitude in Figure $\mathbf{6}$ and the efficiency spectrum in Figure 7 respectively.

In Figure 8, the properties of crystalline PolySilicon are exhibited. The three graphs are the optimized efficiency, $\mathrm{N}$ and $\mathrm{K}$ and the reflectivity's real and imaginary parts. In Figures 9 and 10, the very different material properties of $\mathrm{SiO}_{2}$ and $\mathrm{Si}_{3} \mathrm{~N}_{4}$ are displayed using the same order of presentation as in PolySilicon (Figure 8).

\section{Summary}

This paper represents a novel methodology to characterize the optical properties of lossy materials employed in

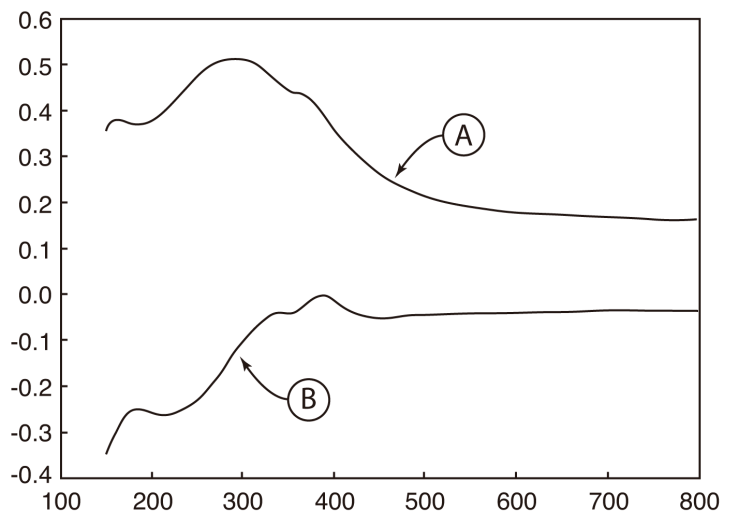

Figure 5. Real (A) and Imaginary (B) $\epsilon_{T o t}(\omega)$ as obtained from the initially measured data of $N$ and $K$ of crystalline titanium di-oxide using 21 Lorentz poles, 3 XLorentz poles and 5 Debye poles.

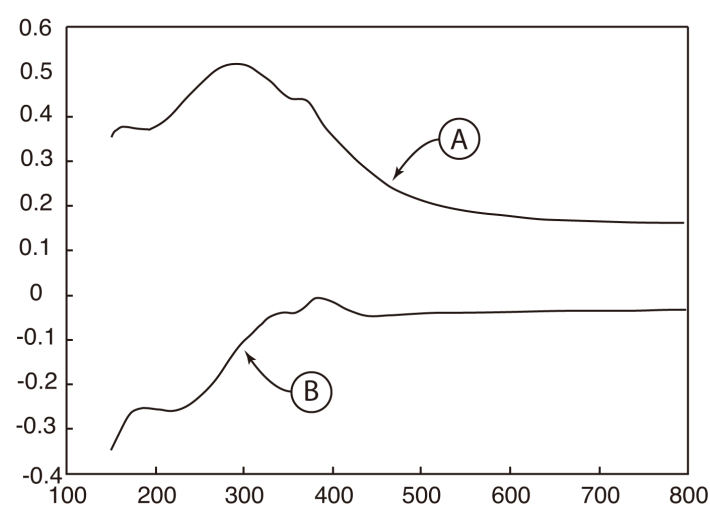

Figure 6. Real (A) and imaginary (B) coefficients of the reflected wave over a thick flat crystalline titanium di-oxide. The graph represents the actual percentage of reflection vs. wave-length in $\mathrm{nm}$ for a pure crystalline titanium di-oxide slab.

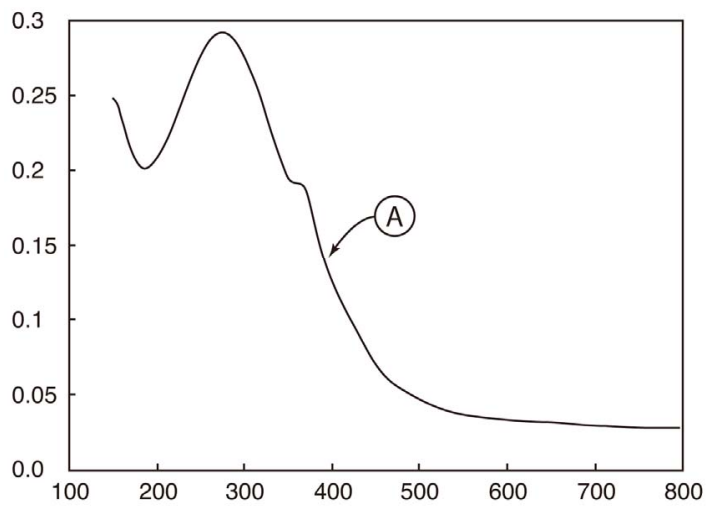

Figure 7. Efficiency Spectrum of planar crystalline titanium di-oxide. The graph represents the actual percentage of reflection vs. wave-length in $\mathrm{nm}$ for a pure crystalline titanium di-oxide slab.
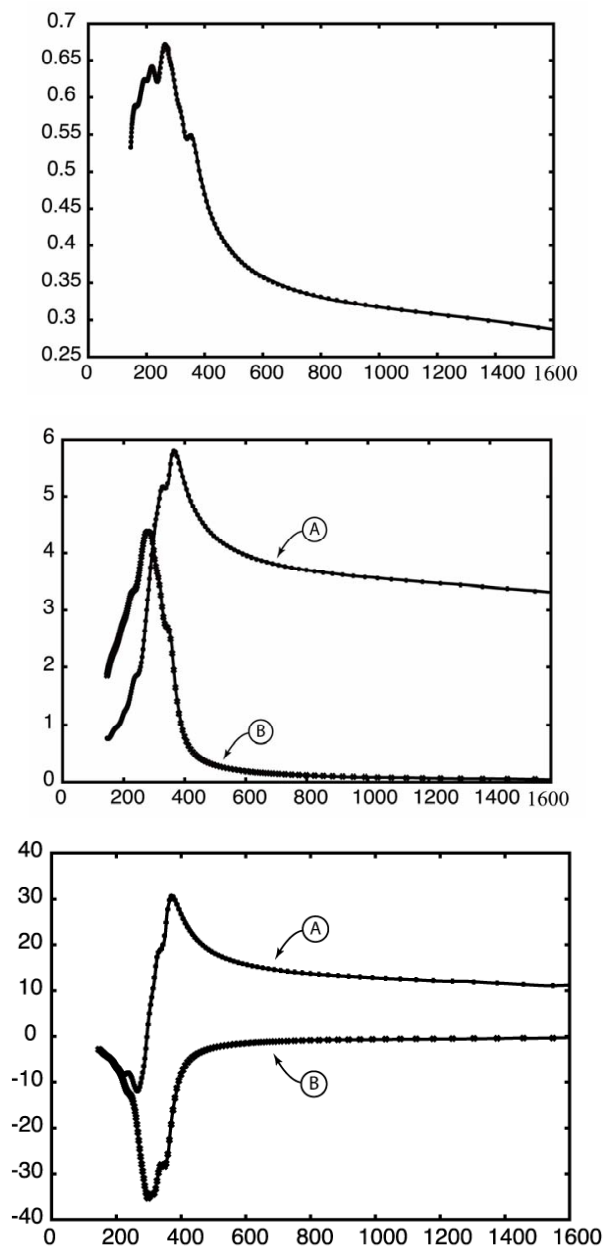

Figure 8. Efficiency, real and imaginary permittivity and reflected field of crystalline PolySilicon.

the microchip and integrated circuit industry. The characterization of the complex permittivity function is represented as sum of terms, each of which preserves causality and undergoes an optimization scheme against real 

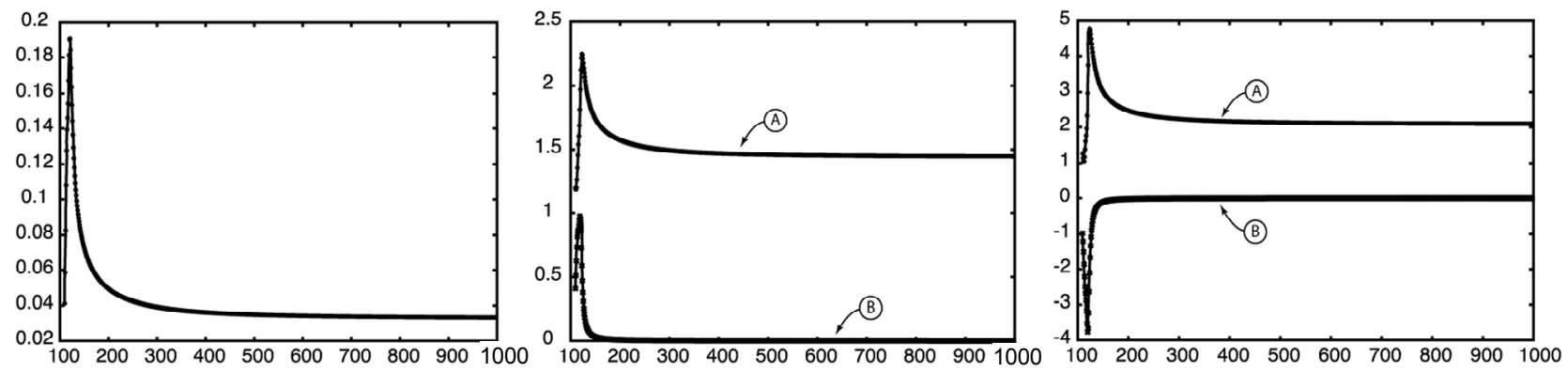

Figure 9. Efficiency, real and imaginary permittivity and reflected field of crystalline $\mathrm{SiO}_{2}$.
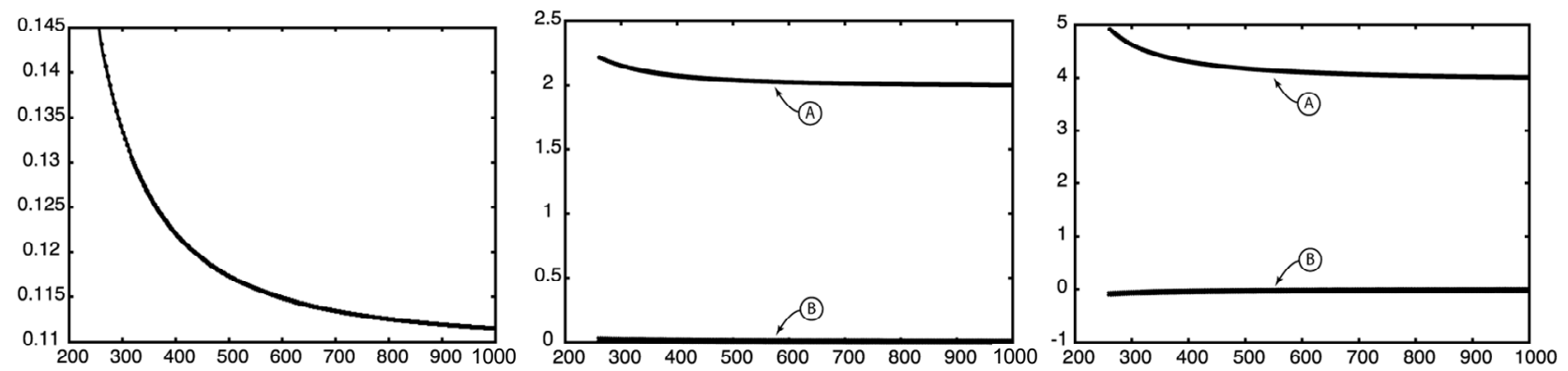

Figure 10. Efficiency, real and imaginary permittivity and reflected field of crystalline $\mathrm{Si}_{3} \mathrm{~N}_{4}$.

measurements via a cost function. The scheme is very fast and efficient, providing a continuous representation of the optical materials outside the range of measurement, thus enabling broadband direct and inverse scattering calculations and predictions.

\section{Acknowledgements}

The authors thank the late D. Gottlieb for many helpful discussions and Andrew Abrahamson for technical assistance. One of us (EB) dedicates this paper to the deceased colleagues and friends Steve A. Orszag, Steve L. Knodle and David Gottlieb.

\section{REFERENCES}

[1] S. Abarbanel, D. Gottlieb and J. S. Hesthaven, "Long Time Behavior of the Perfectly Matched Layer Equations in Computational Electromagnetics," Journal of Scientific
Computing, Vol. 17, No. 1-4, 2002, pp. 405-422. doi:10.1023/A:1015141823608

[2] W. H. Press, B. P. Flannery, S. A. Teukolsky and W. T. Vettering, "Numerical Recipes," Cambridge University Press, Cambridge, 1986.

[3] E. Barouch, S. L. Knodle and S. A. Orszag, "A Broadband Electromagnetic Direct and Inverse Scatterin of Nonlinear Lossy Targets," SPIE Journals, 2013, (in press).

[4] M. Born and E. Wolf, "Principles of Optics," Pergamon Press, Oxford, 1987.

[5] E. M. Lifshitz, "The Theory of Molecular Attractive Forces between Solids," Soviet Physics, Vol. 2, 1956, pp. 73 83 .

[6] F. Pinto, "Engine Cycle of an Optically Controlled Vacuum Energy Transducer," Physical Review B, Vol. 60, 1999, pp. 14740-14755. doi:10.1103/PhysRevB.60.14740 